\title{
UNIFORM CONTRACTIVITY IN WASSERSTEIN METRIC FOR THE ORIGINAL 1D KAC'S MODEL
}

\author{
MAXIME HAURAY
}

\begin{abstract}
We study here a very popular 1D jump model introduced by Kac: it consists of $N$ velocities encountering random binary collisions at which they randomly exchange energy. We show the uniform (in $N$ ) exponential contractivity of the dynamics in a non-standard Monge-Kantorovich-Wasserstein: precisely the MKW metric of order 2 on the energy. The result is optimal in the sense that for each $N$, the contractivity constant is equal to the $L^{2}$ spectral gap of the generator associated to Kac's dynamic. As a corollary, we get an uniform but non optimal contractivity in the MKW metric of order 4. We use a simple coupling that works better that the parallel one. The estimates are simple and new (to the best of our knowledge).
\end{abstract}

\section{INTRODUCTION AND RESULTS}

We study here the following model, introduced by Kac in [5] and intensively studied since (we refer to the review paper [2] or to [7] for more details). The velocities $\left(V_{i, t}\right)_{i \leq \mathbb{N}}, t \geq 0 \in \mathbb{R}^{N}$ of the $N$ particles follows the above dynamics: For $N(N-1) / 2$ independent Poisson random measures $\left(\Pi_{i, j}\right)_{i<j}$ on $\mathbb{R}^{+} \times \mathbb{R} / 2 \pi \mathbb{Z}$ with the same intensity measure $\frac{1}{\pi(N-1)} d t \times d \theta$,

$$
V_{i, t}=V_{i, 0}+\sum_{j \neq i} \int_{0}^{t} \int_{-\pi}^{\pi}\left[\left(V_{i, t^{-}} \cos \theta+V_{j, t^{-}} \sin \theta\right)-V_{i, t^{-}}\right] \Pi_{i, j}(d t, d \theta),
$$

where for $i>j$, the $\Pi_{i, j}=\Pi_{j, i} \circ s$, with $s(\theta)=-\theta$. The normalization in the intensity measure is chosen in order to be consistent with previous work on the subject, in particular [1]. It means that a particle encounters in average two collisions per unit of time. Existence of uniqueness of solutions to that kind of jump process is very standard: for almost all realizations there is at most one collision at each time, i.e. $\quad \sum_{i<j} \Pi_{i, j}(\{t\} \times(-\pi, \pi]) \leq 1$ for all $t \geq 0$, so that the unique solution is easy to construct.

A less rigorous but more simple description of the dynamics is the following: at independent random times $\tau_{i, j}$ (for $i \neq j$ ) with exponential law $\mathcal{E}(1)$, the particles $i$ and $j$ exchange their kinetic energy and jump in the following way (with $t=\tau_{i, j}$ ):

$$
\left(\begin{array}{c}
V_{i, t^{-}} \\
V_{j, t^{-}}
\end{array}\right) \stackrel{\text { collision }}{\longrightarrow}\left(\begin{array}{l}
V_{i, t} \\
V_{j, t}
\end{array}\right)=\left(\begin{array}{c}
V_{i, t^{-}} \cos \theta+V_{j, t^{-}} \sin \theta \\
-V_{i, t^{-}} \sin \theta+V_{j, t^{-}} \cos \theta
\end{array}\right)
$$

where $\theta$ is a r.v. with uniform law on $\mathbb{R} / 2 \pi \mathbb{Z}$, independent of everything else.

2010 Mathematics Subject Classification. 82C40 Kinetic theory of gases and 76P05 Rarefied gases flow, Boltzmann equation and 60J75 Jump processes.

Key words and phrases. Landau equation, Uniqueness, Stochastic particle systems, Propagation of Chaos, Fisher information, Entropy dissipation. 
It is possible to use parallel coupling (same collision and same angle for each collision) between two processes $\left(V_{i, t}\right)_{i \leq \mathbb{N}}$ and $\left(W_{i, t}\right)_{i \leq \mathbb{N}}$ solution of (1). It leads to a result similar to the one obtained by Tanaka 9 for the Boltzmann-Kac model: the coupled evolution is contractive in the MKW metric of order 2.

However, in 1D there is a better coupling: roughly it is related to the fact that optimal coupling between two uniform measures on (distinct) circles of center 0 and different radius is obtained when we preserve the angles. More precisely, we introduce the angle $\alpha \in(-\pi, \pi] \operatorname{such}$ that $\left(V_{i, t^{-}}, V_{j, t^{-}}\right)=\sqrt{V_{i, t^{-}}^{2}+V_{j, t^{-}}^{2}}(\cos \alpha, \sin \alpha)$. Then, the velocities after collision read

$$
\left(V_{i, t^{-}}, V_{j, t^{-}}\right) \stackrel{\text { collision }}{\longrightarrow}\left(V_{i, t}, V_{j, t}\right)=\sqrt{V_{i, t^{-}}^{2}+V_{j, t^{-}}^{2}}(\cos (\alpha-\theta), \sin (\alpha-\theta))
$$

Since the $\theta$ has uniform law and is independent of everything else, so is $\alpha-\theta$. A interesting consequence is that the solution to (1) has exactly the same law than the (unique) solution $\left(\widetilde{V}_{i, t}\right)_{i \leq N}$ to the process

$$
\widetilde{V}_{i, t}=V_{i, 0}+\sum_{j \neq i} \int_{0}^{t} \int_{-\pi}^{\pi}\left[\sqrt{\widetilde{V}_{i, t^{-}}^{2}+\widetilde{V}_{j, t^{-}}^{2}} \cos \theta-\widetilde{V}_{i, t^{-}}\right] \Pi_{i, j}(d t, d \theta)
$$

where this time $\Pi_{i, j}$ for $i>j$ is defined as $\Pi_{i, j}:=\Pi_{j, i} \circ r$, with $r(\theta)=\theta+\frac{\pi}{2}$ (in order to transform cosinus in sinus). Remark that since the angle $\alpha$ introduce above depends continuously on the couple $\left(V_{i, t^{-}}, V_{j, t^{-}}\right)$, so that there is no measurability issue when we pass from the first formulation (1) to the second one (2).

Since the two systems are equivalent in law, we now focus only on the second one, and drop the tilde in the notation. Using parallel coupling with two systems solutions to (2), we obtain an "optimal" contractivity result:

Theorem 1. Assume that $\left(V_{i, t}\right)_{i \leq N}$ and $\left(W_{i, t}\right)_{i \leq N}$ are two solutions to (2) constructed on the same probability space, with normalized energy : $\sum_{i}\left(V_{i, 0}\right)^{2}=$ $\sum_{i}\left(W_{i, 0}\right)^{2}=N$ a.s.. Then

$$
\mathbb{E}\left[\frac{1}{N} \sum_{i=1}^{N}\left[\left(V_{i, t}\right)^{2}-\left(W_{i, t}\right)^{2}\right]^{2}\right]=e^{-\lambda_{N} t} \mathbb{E}\left[\frac{1}{N} \sum_{i=1}^{N}\left(V_{i, 0}^{2}-W_{i, 0}^{2}\right)^{2}\right],
$$

with $\lambda_{N}=\frac{1}{2}+\frac{3}{2(N-1)}=\frac{1}{2} \frac{N+2}{N-1} \geq \frac{1}{2}$.

Remark 2. Remark that $\lambda_{N}$ is also the spectral gap in $L^{2}$ of the associated generator, which was explicitly computed in [1, 6]. But according to [3, Theorem 2.42], the spectral gap is a bound by above on any contraction rate than could be obtain by coupling. So in that sense, the contraction rate $\lambda_{N}$ obtained above is optimal.

Moreover, the fact that our coupling cost $\left(v^{2}-w^{2}\right)^{2}$ is of order four in the velocity is related to the fact that the eigenfunction associated to the eigenvalue $-\lambda_{N}$ of the associated generator is a polynomial of degree four: precisely $\sum_{i} v_{i}^{4}-c$, for some constant $c$.

This optimal result with a non standard metric (the MKW metric of order 2 on the energy) implies a non optimal result in MKW metric of order 4: 
Corollary 3. Under the same assumptions than in Theorem 1, we have

$$
\begin{aligned}
\mathbb{E}\left[\frac{1}{N} \sum_{i=1}^{N}\left(V_{i, t}-W_{i, t}\right)^{4}\right] \leq e^{-\lambda_{N} t} \mathbb{E}\left[\frac { 1 } { N } \sum _ { i = 1 } ^ { N } \left(V_{i, 0}^{2}\right.\right. & \left.\left.-W_{i, 0}^{2}\right)^{2}\right] \\
& +e^{-t} \mathbb{E}\left[\frac{1}{N} \sum_{i=1}^{N}\left(V_{i, 0}-W_{i, 0}\right)^{4}\right] .
\end{aligned}
$$

The question whether these results could be extended to the case where the angle $\theta$ are not uniform, or to larger dimension remains open. We refer to the works of Mischler and Mouhot [7] and Rousset [8] for the best results in higher dimension.

In order to obtain propagation of chaos towards the limit nonlinear jump process, the strategy used in [4] could be applied.

Extension to the nonlinear limit model. A similar result could be obtained on the limit nonlinear jump process

$$
V_{t}=V_{0}+\int_{0}^{t} \int_{-\pi}^{\pi}\left[\left(V_{t^{-}} \cos \theta+F_{\mathcal{L}\left(V_{t^{-}}\right)}^{-1}(u) \sin \theta\right)-V_{t^{-}}\right] \Pi(d t, d \theta, d u),
$$

where $\Pi$ is a Poisson random measure on $\mathbb{R}^{+} \times \mathbb{R} / 2 \pi \mathbb{Z} \times[0,1]$ with intensity measure $\frac{1}{\pi(N-1)} d t \times d \theta \times d u$, and $F_{\mathcal{L}\left(V_{t^{-}}\right)}^{-1}$ stands for the pseudo-inverse of the cumulative distribution function of $V_{t^{-}}$.

Similarly, that process is equivalent (in law) to

$$
V_{t}=V_{0}+\int_{0}^{t} \int_{-\pi}^{\pi}\left[\sqrt{V_{t^{-}}^{2}+\left(F_{\mathcal{L}\left(V_{t^{-}}\right)}^{-1}(u)\right)^{2}} \cos \theta-V_{t^{-}}\right] \Pi(d t, d \theta, d u),
$$

and we have a similar result of contraction:

Theorem 4. Assume that $\left(V_{t}\right)_{t \geq 0}$ and $\left(W_{t}\right)_{t \geq 0}$ are two solutions of (41) constructed with the same PRM П. Then,

$$
\mathbb{E}\left[\left(V_{t}^{2}-W_{t}^{2}\right)^{2}\right]=e^{-\frac{t}{2}} \mathbb{E}\left[\left(V_{0}^{2}-W_{0}^{2}\right)^{2}\right] .
$$

We will not prove that theorem, since the proof is really similar to the one of Theorem 1. A similar corollary on the decrease of the order four MKW distance could be stated.

\section{Proofs}

Proof of Theorem 1. In order to simplify the writing, we introduce notations for the energy (up to a factor 2): $E_{i, t}:=\left(V_{i, t}\right)^{2}$ and $F_{i, t}:=\left(W_{i, t}\right)^{2}$. Then, both energy vectors satisfy the following stochastic process:

$$
E_{i, t}=E_{i, 0}+\sum_{j \neq i} \int_{0}^{t} \int_{-\pi}^{\pi}\left[\left(E_{i, t^{-}}+E_{j, t^{-}}\right) \cos ^{2} \theta-E_{i, t^{-}}\right] \Pi_{i, j}(d t, d \theta),
$$

where $\Pi_{i, j}:=\Pi_{j, i} \circ r$ for $i>j$, with $r(\theta)=\theta+\frac{\pi}{2}$, as before. The $\left(F_{i, t}\right)_{i \leq N, t \geq 0}$ satisfies the same equations, and so does $G_{i, t}:=E_{i, t}-F_{i, t}$ by linearity of (5).

Next, since the total energy is preserved by the dynamics, we have almost surely for all $t>0$ :

$$
\frac{1}{N} \sum_{i=1}^{N} E_{i, t}=\frac{1}{N} \sum_{i=1}^{N} F_{i, t}=1, \quad \frac{1}{N} \sum_{i=1}^{N} G_{i, t}=0 .
$$


We are interested in the evolution of $\frac{1}{N} \sum_{i} G_{i, t}^{2}$. But the process $\left(G_{i, t}^{2}\right)_{i \leq N, t \geq 0}$ satisfies

$$
G_{i, t}^{2}=G_{i, 0}^{2}+\sum_{j \neq i} \int_{0}^{t} \int_{-\pi}^{\pi}\left[\left(G_{i, t^{-}}+G_{j, t^{-}}\right)^{2} \cos ^{4} \theta-G_{i, t^{-}}^{2}\right] \Pi_{i, j}(d t, d \theta) .
$$

It implies that

$\left.\frac{d}{d t} \mathbb{E}\left[\frac{1}{N} \sum_{i} G_{i, t}^{2}\right]=\mathbb{E}\left[\frac{2}{N(N-1)} \sum_{i<j}\left(\left(G_{i, t}+G_{j, t}\right)^{2}\left(\cos ^{4} \theta+\sin ^{4} \theta\right)-G_{i, t}^{2}-G_{j, t}\right)^{2}\right)\right]$.

Since $\frac{1}{2 \pi} \int_{-\pi}^{\pi}\left(\cos ^{4} \theta+\sin ^{4} \theta\right) d \theta=\frac{3}{4}$, it also reads

$$
\frac{d}{d t} \mathbb{E}\left[\frac{1}{N} \sum_{i}\left(G_{i, t}\right)^{2}\right]=-\frac{1}{2} \mathbb{E}\left[\frac{1}{N} \sum_{i}\left(G_{i, t}\right)^{2}\right]+\frac{3}{2 N(N-1)} \mathbb{E}\left[\sum_{i \neq j} G_{i, t} G_{j, t}\right] .
$$

We emphasize that we have replaced the sum on $\{i<j\}$ by a sum on $\{i \neq g\}$. But

$$
\sum_{i \neq j} G_{i, t} G_{j, t}=\left(\sum_{i} G_{i, t}\right)^{2}-\sum_{i}\left(G_{i, t}\right)^{2}=-\sum_{i}\left(G_{i, t}\right)^{2},
$$

thanks to the conservation of energy (6). So we finally have

$$
\frac{d}{d t} \mathbb{E}\left[\frac{1}{N} \sum_{i}\left(G_{i, t}\right)^{2}\right]=-\left(\frac{1}{2}+\frac{3}{2(N-1)}\right) \mathbb{E}\left[\frac{1}{N} \sum_{i}\left(G_{i, t}\right)^{2}\right],
$$

and the conclusion follows.

Proof of Corollary [3. In equation (2), we see that the sign of $V_{i, t}$ after a collision is the one of $\cos \theta$, where $\theta$ is the angle used in that collision, (and the $\operatorname{sign}$ of $V_{j, t}$ is the one of $\sin \theta)$. Since the same angle are used during the evolution of $\left(W_{i, t}\right)_{i \leq N, t \geq 0}$, it implies than $V_{i, t}$ and $W_{i, t}$ have the same sign after the first collision involving the $i$-th particle. And if they have the same sign,

$$
\left(V_{i, t}-W_{i, t}\right)^{4} \leq\left(V_{i, t}-W_{i, t}\right)^{2}\left(V_{i, t}+W_{i, t}\right)^{2}=\left(V_{i, t}^{2}-W_{i, t}^{2}\right)^{2} .
$$

We call $\tau_{i}$ the stopping time defined has the time of the first collision involving $i$. We get

$$
\begin{aligned}
\mathbb{E}\left[\frac{1}{N} \sum_{i=1}^{N}\left(V_{i, t}-W_{i, t}\right)^{4}\right] \leq \mathbb{E}\left[\frac{1}{N} \sum_{i=1}^{N}\left(V_{i, t}^{2}-W_{i, t}^{2}\right)^{2} \mathbb{I}_{t \geq \tau_{i}}\right] & \\
+ & \mathbb{E}\left[\frac{1}{N} \sum_{i=1}^{N}\left(V_{i, t}-W_{i, t}\right)^{4} \mathbb{I}_{t \leq \tau_{i}}\right]
\end{aligned}
$$

The first term in the r.h.s. is bounded thanks to the previous result (the indicator function is bounded by 1). To bound the second term, remark that each $\tau_{i}$ is independent of the initial condition and as exponential law $\mathcal{E}(1)$, so that

$$
\begin{aligned}
\mathbb{E} & {\left[\frac{1}{N} \sum_{i=1}^{N}\left(V_{i, t}-W_{i, t}\right)^{4} \mathbb{I}_{t \leq \tau_{i}}\right]=\frac{1}{N} \sum_{i=1}^{N} \mathbb{E}\left[\left(V_{i, 0}-W_{i, 0}\right)^{4} \mathbb{I}_{t \leq \tau_{i}}\right] } \\
& =\frac{1}{N} \sum_{i=1}^{N} \mathbb{E}\left[\left(V_{i, 0}-W_{i, 0}\right)^{4}\right] \mathbb{P}\left(\tau_{i} \geq t\right)=e^{-t} \mathbb{E}\left[\frac{1}{N} \sum_{i=1}^{N}\left(V_{i, 0}-W_{i, 0}\right)^{4}\right]
\end{aligned}
$$


The conclusion follows.

Acknowledgements The authors would like to thank Matthias Rousset and Alexandre Gaudillière for very stimulating discussion about that problem. In particular the proof of Corollary 3 is due to the latter.

\section{REFERENCES}

[1] E. Carlen, M. Carvalho, and M. Loss, Determination of the spectral gap for Kacs master equation and related stochastic evolution, Acta Math., 191, 1-54 (2003).

[2] E. Carlen, M. Carvalho, and M. Loss, Kinetic theory and the Kac master equation. Entropy and the quantum II, 1-20, Contemp. Math., 552, Amer. Math. Soc., Providence (2011).

[3] M.-F. Chen, Eigenvalues, inequalities, and ergodic theory, Probability and its Applications (New York), Springer-Verlag, London (2005).

[4] R. Cortez, J. Fontbona, Quantitative propagation of chaos for generalized Kac particle systems, to appear in Ann. Appl. Probab., arXiv:1406.2115

[5] M. Kac, Foundations of kinetic theory, In Proceedings of the Third Berkeley Symposium on Mathematical Statistics and Probability, 1954-1955, vol. III, 171-197, University of California Press, Berkeley and Los Angeles (1956).

[6] D.K. Maslen, The eigenvalues of Kac's master equation, Math. Z., 243, 291-331 (2003).

[7] S. Mischler, C. Mouhot, Kac's program in kinetic theory, Invent. Math. 193, 1-147 (2013).

[8] M. Rousset, A $N$-uniform quantitative Tanaka's theorem for the conservative Kac's $N$-particle system with Maxwell molecules, arXiv:1407.1965 (2014).

[9] H. Tanaka, Probabilistic treatment of the boltzmann equation of maxwellian molecules, $\mathrm{Z}$. Wahrsch. Verw. Gebiete, 46, 67-105 (1978).

M. Hauray: Institut de Mathématiques de Marseille, Université D’Aix-Marseille, CNRS UMR 7353 et École Centrale Mrseille.

E-mail address: maxime.hauray@univ-amu.fr 